\title{
A Complex Variational Approach for Single Loaded/Unloaded Hole in Finite Laminated Plate under General In-Plane Loads
}

\author{
Y. Xiong \\ Structures, Materials and Propulsion Laboratory \\ Institute for Aerospace Research, National Research Council \\ Ottawa, Canada KIA OR6
}

\section{ABSTRACT}

A complex variational approach is developed in this paper for calculating the stresses around a single pinloaded or unloaded hole in a composite laminate of finite geometry under general in-plane loads. In the loaded hole case, the pin is assumed to be infinitely rigid and the friction on the contact region of the hole edge is ignored. The pin load is determined from the equilibrium condition with the external applied loads and is assumed to be in a cosine distribution over one half of the hole edge. Displacement-based variational formulations are derived for both unloaded and pinloaded hole cases. Two complex stress potentials in the two-dimensional anisotropic elasticity with undetermined constants are used as trial functions of the formulations. The stress and displacement components expressed in terms of these two complex potentials satisfy the equilibrium equations and compatibility relationship in the domain. The variational formulations derived involve boundary integrations only along the interior and exterior edges of the laminate. As a result, the boundary effects are automatically accounted for and correction factors are not required for plates of finite geometry. Through the minimisation of the variational functionals, a set of linear algebraic equations are derived and solved for the constants in the complex potentials selected. The stress concentrations at the hole edges as well as the global stress distribution are then calculated. A convergence study is conducted to verify the complex variational approach developed. Comparisons between the present results and those from closed-form solutions and finite element analysis are
\end{abstract}

made to demonstrate the accuracy and efficiency of the present approach.

\section{INTRODUCTION}

Advanced composite materials have been used increasingly in aerospace structures. The stress concentrations in a composite laminate with a notch, loaded or unloaded, are important factors to be considered in designing composite structures. Stress analysis models are needed to determine the stress concentrations accurately and to predict the failure of laminated plates with a loaded/unloaded hole. There has been extensive work in the literature devoted to the analysis of loaded holes such as mechanically fastened joints, as reviewed by Oplinger $/ 8 /$. However, most of the work in the literature deals with uni-directional loading only. In many cases, such as a laminate with multiple holes, the stress state in the surrounding area of each hole will not be uni-directional due to the interactions. In such cases, general in-plane loads should be considered in order to examine the effects of bi-axial, bypass, as well as shear stresses on the performance of the notched component. The stress analysis of composite laminates with loaded and unloaded holes under general in-plane loads represents a challenging research topic of composite structures.

The two-dimensional finite element method is by far the most common approach used for the stress analysis of composite laminates with fastener holes (see, e.g., Waszczak and Cruse /9/, Crews, Hong and Raju /3/, and Chang, Scott and Springer $/ 2 /$ ). While this numerical 
technique can handle various geometry and general loading conditions, it is not ideal for parametric study or optimum design. In addition, the peak stresses around the notch in the loaded hole case are sensitive to the mesh size used in the analysis.

For design purposes, the closed-form solutions following Lekhnitskii /6/ is an efficient technique (see de Jong $/ 4 /$ and Zhang and Ueng $/ 11 /$ ). This method is based on an infinite plate assumption. Finite width correction factors are required when applying the closed-form solutions to finite sized plates (Garbo and Ogonowski /5/). These correction factors, in most cases, are developed based on finite element analyses. This requirement of using a numerical technique to generate correction factors makes the Lekhnitskii's complex potential method less suitable for designing complex joint configurations. In addition, the closed-form solutions are valid for pure bearing loading only.

The boundary collocation method, originally introduced for crack problems, e.g., Bowie and Neal $/ 1 /$, eliminates the requirement for correction factors, e.g., Madenci and Ileri $77 \%$ This approach involves a conformal mapping and a least-squares scheme. Although the computational simplicity of the boundary collocation method is attractive, the results are sensitive to the selections of both the stress functions and the locations of boundary points. As a result, convergence to incorrect values is possible if the selections are inappropriate. To overcome the drawbacks of the boundary collocation method, a complex variational approach has recently been developed for the stress analysis of finite composite laminates with loaded and unloaded holes under a uni-directional loading condition (Xiong $/ 10 /$ ). In this approach, the boundary conditions are satisfied through a variational statement rather than only on a set of discrete boundary points as in Madenci and Ileri $/ 7 /$.

The present paper discusses part of the research work of a program on Biaxial/Bypass Behavior of Composites, which is funded by the Department of National Defense of Canada and is in collaboration with CAE Aviation Inc. The objective of the program is to develop PC-based software for use by field engineers to design a patch repair involving multiple fasteners. Therefore a relatively simple but accurate analytical approach, rather than a sophisticated finite element method, is required. The complex variational approach (Xiong $/ 10 /$ ) is such a method because it has the advantages of both variational methods to deal with finite boundaries and the complex variable theory to have closed-form solutions in the domain. An extension of the complex variational approach is made in this paper in order to calculate the stresses around an unloaded or pin-loaded hole in a finite laminated plate under general biaxial/bypass loading conditions. The establishment of the new formulation and the analytical results obtained are presented in this paper. The accuracy and efficiency of the present approach are verified by comparison of the results to those from the closed-form solutions in an infinite plate case and the finite element analysis in finite plate cases.

\section{STATEMENT OF PROBLEM}

The problem under study is a rectangular laminated plate of length $L$ and width $W$ containing a centrally located hole with radius $a$, as shown in Figure 1. This is a two-dimensional problem of an anisotropic plate. The central hole may be either unloaded or pin-loaded. The plate is subjected to general in-plane loads, which are distributed along its four exterior edges. The applied loads are self-balanced in the unloaded hole case and are in equilibrium with the pin load in the pin-loaded hole case. The applied edge loads are shown in barred symbols in Figure 1. Also, the following assumptions are made for the sake of simplicity: (i) the pin is infinitely rigid and frictionless; (ii) the pin load is in a cosine distribution over one half of the hole edge; (iii) the resultant of the distributed normal loads is located at the mid-span of the respective edges; and (iv) the shear loads along the four exterior edges are self-balanced and the contribution by the shear loads to the pin load is ignored.

Assumptions (i) and (ii) are made for the purpose of simplicity in discussion since the first step of the theoretical work is to make sure that the variational formulation would work. Numerous researchers have used such assumptions. With a modification in the 


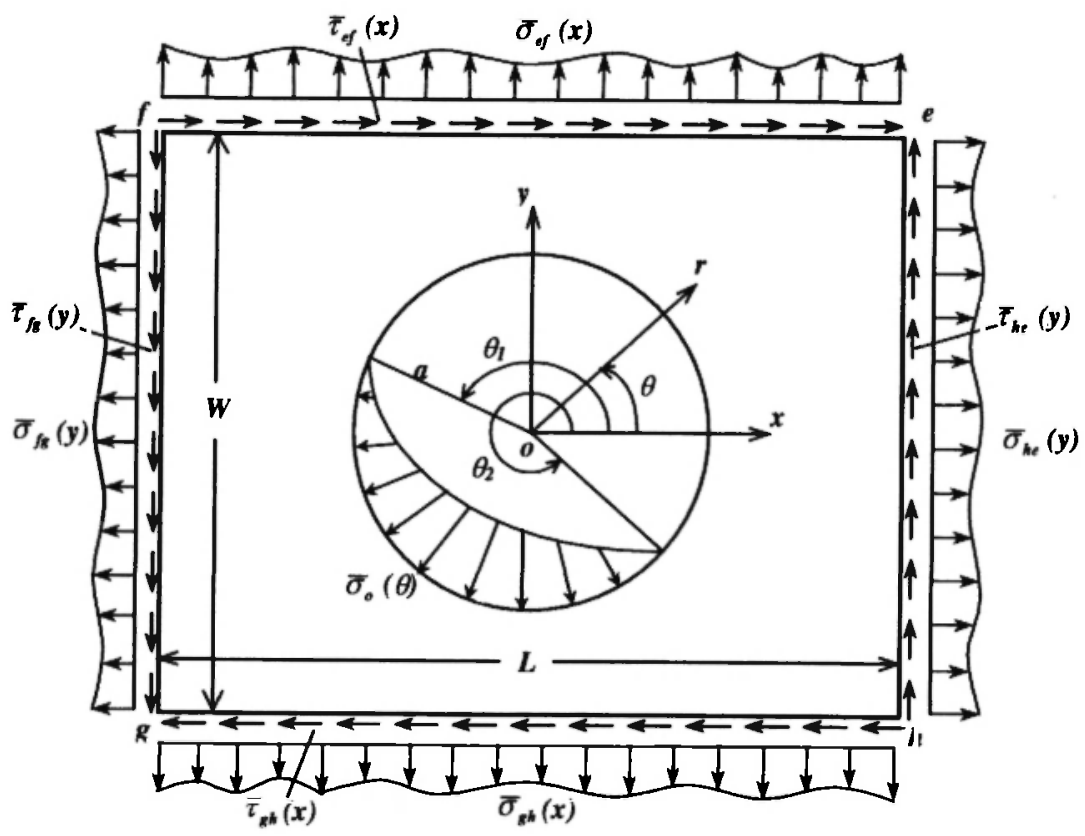

Fig. 1: A pin-loaded hole under general in-plane loads.

formulation, other types of assumption can be adopted by the approach as in Xiong /10/.

A global co-ordinate system, $x-y$, is employed which is located at the centre of the plate. The corresponding stress, strain and displacement components in the plate are denoted as $\sigma_{x}, \sigma_{y}, \tau_{x y}, \varepsilon_{x}, \varepsilon_{y}, \gamma_{x y}, u$, and $v$, respectively. For the purpose of convenience, a polar system, $r-\theta$, is used on the periphery of the hole with the $r$-axis being the outer normal direction and $\theta=0$ being the $x$ axis such that:

$$
x=a \cos \theta, y=a \sin \theta, 0<\theta<2 \pi
$$

The transformations of the stress and displacement components between the two co-ordinate systems are as follows:

$$
\begin{aligned}
& \sigma_{r}==\sigma_{x} \cos ^{2} \theta+\sigma_{y} \sin ^{2} \theta+2 \tau_{x y} \cos \theta \sin \theta \\
& \sigma_{\theta}=\sigma_{x} \sin ^{2} \theta+\sigma_{y} \cos ^{2} \theta+2 \tau_{x y} \cos \theta \sin \theta \\
& \tau_{r \theta}=\left(-\sigma_{x}+\sigma_{y}\right) \cos \theta \sin \theta+\tau_{x y}\left(\cos ^{2} \theta-\sin ^{2} \theta\right)
\end{aligned}
$$

and

$$
u_{r}=u \cos \theta+v \sin \theta, \quad u_{\theta}=-u \sin \theta+v \cos \theta
$$

The problem is to find solutions for the stress and displacement components, which satisfy the equilibrium and compatibility equations in the plate. These equations are written as:

$$
\frac{\partial \sigma_{r}}{\partial x}+\frac{\partial \tau_{x y}}{\partial y}=0, \quad \frac{\partial \tau_{x y}}{\partial x}+\frac{\partial \sigma_{v}}{\partial y}=0
$$

and

$$
\frac{\partial^{2} \varepsilon_{x}}{\partial y^{2}}+\frac{\partial^{2} \varepsilon_{y}}{\partial x^{2}}-\frac{\partial^{2} \gamma_{x y}}{\partial x \partial y}=0
$$

In addition, the solutions must satisfy the boundary conditions along all exterior and interior edges. The boundary conditions for the exterior edges can be expressed as: 


$$
\begin{array}{r}
\sigma_{y}(x, y)=\bar{\sigma}_{e f}(x), \tau_{x y}(x, y)=\bar{\tau}_{e f}(x) \\
\text { along edge } e-f \text { at } y=W / 2 \\
\sigma_{y}(x, y)=\bar{\sigma}_{f g}(y), \tau_{x y}(x, y)=\bar{\tau}_{f g}(y) \\
\text { along edge } f-g \text { at } x=-L / 2 \\
\sigma_{y}(x, y)=\bar{\sigma}_{g h}(x), \tau_{x y}(x, y)=\bar{\tau}_{g h}(x) \\
\text { along edge } g-h \text { at } y=-W / 2 \\
\sigma_{y}(x, y)=\bar{\sigma}_{h e}(y), \tau_{x y}(x, y)=\bar{\tau}_{h e}(y) \\
\text { along edge } h-e \text { at } x=L / 2
\end{array}
$$

The boundary conditions along the interior hole edge are different for unloaded and pin-loaded hole cases. In the former case the total hole edge is traction free, while in the latter case, the pin load is applied towards the hole, causing one half of the hole edge to be in compression. The boundary conditions for unloaded and pin-loaded cases can be written, respectively, as:

For unloaded hole:

$$
\sigma_{r}(a, \theta)=\tau_{r \theta}(a, \theta)=0, \quad 0<\theta<2 \pi
$$

For pin-loaded hole:

$$
\begin{aligned}
& \sigma_{r}(a, \theta)=\tau_{r \theta}(a, \theta)=0, \quad \theta_{2}-2 \pi \leq \theta \leq \theta_{1} \\
& \gamma_{r}(a, \theta)=\bar{\sigma}_{o}(\theta) \text {, } \\
& \left\{\tau_{r \theta}(a, \theta)=0\right. \text {, }
\end{aligned}
$$

where the two angles $\theta_{1}$ and $\theta_{2}$ define the contact region between the pin and the hole edge as $\theta_{1}-\dot{\theta}_{2}$ which is assumed to be one half circle in the present work. The compression by the pin over the contact region is assumed to be in a cosine distribution as:

$$
\left.\bar{\sigma}_{o}(\theta)=-\frac{2 P_{f}}{\pi a} \cos \frac{\theta-\theta_{1}}{\theta_{2}-\theta_{1}} \pi+\frac{\pi}{2}\right), \theta_{1} \leq \theta \leq \theta_{2}
$$

where $P_{f}$ is the pin load. Based on the equilibrium consideration with the external applied loads, the pin load and the contact region can be determined, for various situations, as:
$\begin{array}{ll}P_{f f}=\stackrel{L}{L / 2}_{L / 2}^{L}\left[\bar{\sigma}_{e f}(x)-\bar{\sigma}_{g h}(x)\right] d x, & L_{L / 2}^{L / 2}\left[\bar{\sigma}_{e f}(x)-\bar{\sigma}_{g h}(x)\right] d x>0 \\ \theta_{1}=\pi, \theta_{2}=2 \pi, & \int_{W / 2}^{W / 2}\left[\bar{\sigma}_{h e}(y)-\bar{\sigma}_{f g}(y)\right] d y=0\end{array}$

$\begin{array}{ll}P_{f}=\int_{L / 2}^{L / 2}\left[\bar{\sigma}_{g h}(x)-\bar{\sigma}_{e f}(x)\right] d x, & \int_{L / 2}^{L / 2}\left[\bar{\sigma}_{e f}(x)-\bar{\sigma}_{g h}(x)\right] d x<0 \\ \theta_{1}=0, \theta_{2}=\pi, & \int_{W / 2}^{W / 2}\left[\bar{\sigma}_{h e}(y)-\bar{\sigma}_{f g}(y)\right] d y=0\end{array}$

$\begin{array}{ll}P_{f}=\int_{W / 2}^{W / 2}\left[\bar{\sigma}_{h e}(y)-\bar{\sigma}_{f g}(y)\right] d y, \text { if } & \int_{L / 2}^{L / 2}\left[\bar{\sigma}_{e f}(x)-\bar{\sigma}_{g h}(x)\right] d x=0 \\ \theta_{1}=\pi / 2, \theta_{2}=3 \pi / 2, & L_{W / 2}^{L V ! ?}\left[\bar{\sigma}_{h e}(y)-\bar{\sigma}_{f g}(y)\right] d y>0\end{array}$

$\begin{array}{ll}P_{f}=\int_{W / 2}^{W / 2}\left[\bar{\sigma}_{f g}(y)-\bar{\sigma}_{h e}(y)\right] d y, & \int_{L / 2}^{L / 2}\left[\bar{\sigma}_{e f}(x)-\bar{\sigma}_{g h}(x)\right] d x=0 \\ \theta_{1}=-\pi / 2, \theta_{2}=\pi / 2, & \\ & \int_{W / 2}^{W / 2}\left[\bar{\sigma}_{h e}(y)-\bar{\sigma}_{f g}(y)\right] d y<0\end{array}$ 


$$
\begin{aligned}
& P_{f}=\sqrt{\left\{\int_{L / 2}^{L / 2}\left[\bar{\sigma}_{e f}(x)-\bar{\sigma}_{g h}(x)\right] d x\right\}^{2}+\left\{\int_{W / 2}^{N / 2}\left[\bar{\sigma}_{h e}(y)-\bar{\sigma}_{f g}(y)\right] d y\right\}^{2}}, \\
& \theta_{1}=\pi / 2+\arctan \left\{\frac{\int_{-i / 2}^{L / 2}\left[\bar{\sigma}_{e f}(x)-\bar{\sigma}_{g h}(x)\right] d x}{L_{W / 2}^{\prime \prime \prime 2}\left[\bar{\sigma}_{h e}(y)-\bar{\sigma}_{f g}(y)\right] d y}\right\}, \theta_{2}=\theta_{1}+\pi \text {, } \\
& \text { if } \quad \int_{L / 2}^{L / 2}\left[\bar{\sigma}_{e f}(x)-\bar{\sigma}_{g h}(x)\right] d x \neq 0, \int_{W / 2}^{W / 2}\left[\bar{\sigma}_{h e}(y)-\bar{\sigma}_{f g}(y)\right] d y>0 \\
& P_{f}=\sqrt{\left\{\int_{-L / 2}^{L / 2}\left[\bar{\sigma}_{e f}(x)-\bar{\sigma}_{g h}(x)\right] d x\right\}^{2}+\left\{\int_{W / 2}^{W / 2}\left[\bar{\sigma}_{h e}(y)-\bar{\sigma}_{f g}(y)\right] d y\right\}^{2}}, \\
& \theta_{1}=-\pi / 2+\arctan \left\{\frac{\left[_{-L / 2}^{L / 2}\left[\bar{\sigma}_{e f}(x)-\bar{\sigma}_{g h}(x)\right] d x\right.}{L_{W / 2}^{\prime \prime / 2}\left[\bar{\sigma}_{h e}(y)-\bar{\sigma}_{f g}(y)\right] d y}\right\}, \theta_{2}=\theta_{1}+\pi, \\
& \text { if } \quad \int_{-L / 2}^{L / 2}\left[\bar{\sigma}_{e f}(x)-\bar{\sigma}_{g h}(x)\right] d x \neq 0, \int_{W / 2}^{W / 2}\left[\bar{\sigma}_{h e}(y)-\bar{\sigma}_{f g}(y)\right] d y<0
\end{aligned}
$$

\section{BASIC RELATIONS OF 2D ANISOTROPIC ELASTICITY}

In the theory of the two dimensional anisotropic elasticity, two complex stress potentials, $\varphi_{1}$ and $\varphi_{2}$, are employed to derive the stress and displacement components. In this way, the equilibrium and compatibility equations (4) and (5) are automatically satisfied in the domain of the plate. The two stress potentials are functions of two complex co-ordinate variables, $z_{1}$ and $z_{2}$, defined as:

$$
Z_{1}=x+\mu_{1}, y, z_{2}=x+\mu_{2} y
$$

where the two complex constants, $\mu_{1}$ and $\mu_{2}$, are the two distinct complex roots with positive imaginary part of the characteristic equation of the laminate. The characteristic equation is written in terms of the in-plane compliance coefficients, $a_{\mathrm{ij}}(\mathrm{i}, \mathrm{j}=1,2,6)$, as:

$a_{11} \mu^{4}-2 a_{16} \mu^{3}+\left(2 a_{12}+a_{66}\right) \mu^{2}-2 a_{26} \mu+a_{22}=0(17)$

The stress components take the following form:

$$
\begin{aligned}
& \sigma_{x}=2 \operatorname{Re}\left[\mu_{1}^{2} \frac{d \varphi_{1}\left(z_{1}\right)}{d z_{1}}+\mu_{2}^{2} \frac{d \varphi_{2}\left(z_{2}\right)}{d z_{2}}\right] \\
& \sigma_{y}:=2 \operatorname{Re}\left[\frac{d \varphi_{1}\left(z_{1}\right)}{d z_{1}}+\frac{d \varphi_{2}\left(z_{2}\right)}{d z_{2}}\right] \\
& \tau_{x y}=-2 \operatorname{Re}\left[\mu_{1} \frac{d \varphi_{1}\left(z_{1}\right)}{d z_{1}}+\mu_{2} \frac{d \varphi_{2}\left(z_{2}\right)}{d z_{2}}\right]
\end{aligned}
$$

and the corresponding displacement components are:

$$
\begin{aligned}
& u=2 \operatorname{Re}\left[p_{1} \varphi_{1}\left(z_{1}\right)+p_{2} \varphi_{2}\left(z_{2}\right)\right] \\
& v=2 \operatorname{Re}\left[q_{1} \varphi_{1}\left(z_{1}\right)+q_{2} \varphi_{2}\left(z_{2}\right)\right]
\end{aligned}
$$

where the two pair of complex constants are defined as:

$$
\begin{aligned}
& p_{k}=a_{11} \mu \frac{2}{t}+a_{12}-a_{16} \mu_{k}, \\
& q_{k}=a_{12} \mu_{k}+\frac{a_{22}}{\mu_{k}}-a_{26}, k=1,2
\end{aligned}
$$

Note that the constants for the rigid body motions are set to zero in Eq. (19) because they do not cause any 
strains in the plate. The stress and displacement components are related to each other by the constitutive equations expressed as:

$$
\begin{aligned}
& \sigma_{x}=A_{11} \frac{\partial u}{\partial x}+A_{12} \frac{\partial v}{\partial y}+A_{16}\left(\frac{\partial u}{\partial y}+\frac{\partial v}{\partial x}\right) \\
& \sigma_{y}=A_{12} \frac{\partial u}{\partial x}+A_{22} \frac{\partial v}{\partial y}+A_{26}\left(\frac{\partial u}{\partial y}+\frac{\partial v}{\partial x} ;\right. \\
& \tau_{x y}=A_{16} \frac{\partial u}{\partial x}+A_{26} \frac{\partial v}{\partial y}+A_{66}\left(\frac{\partial u}{\partial y}+\frac{\partial v}{\partial x}\right)
\end{aligned}
$$

where $A_{\mathrm{ij}}(\mathrm{i}, \mathrm{j}=1,2,6)$ are the in-plane stiffness coefficients, that is $\left[A_{\mathrm{ij}}\right]^{-1}$ is equal to $\left[a_{\mathrm{ij}}\right]$.

\section{COMPLEX VARIATIONAL FORMULATIONS}

Since the stress and displacement components expressed in terms of the two stress potentials as in Eqs. (18) and (19) satisfy the equilibrium and compatibility equations in the domain of the plate, the remaining task is to find appropriate stress potentials so that the respective boundary conditions can be satisfied. For this purpose, variational formulations are established in this section for both unloaded and pin-loaded cases. Note that the difference between the unloaded and pin-loaded cases exists only with the boundary conditions along the hole edge. As a matter of fact, the unloaded hole case is a special loaded hole case when the pin load is zero.

For the unloaded hole case, all boundary conditions are traction boundary conditions. Therefore a displacement-based variational formulation can be used with the total potential energy functional written as: where $\Omega$ denotes the domain of the plate. Integrating Eq. (22) by parts and using the constitutive relations in Eq. (21), the first order variation of the above energy functional with respect to $u$ and $v$, respectively, is derived and written as:

$$
\begin{aligned}
\delta \Pi_{u n t}= & \int_{\Omega}\left(-\left(\frac{\partial \sigma_{x}}{\partial x}+\frac{\partial \tau_{x y}}{\partial y}\right) \delta u-\left(\frac{\partial \tau_{x y}}{\partial x}+\frac{\partial \sigma_{y}}{\partial y}\right) \delta v\right) d x d y \\
& + \text { Boundary Terms }
\end{aligned}
$$

where the boundary integrations are not written explicitly for the simplicity. Note that all the stress and displacement components are actually related to the two stress potentials, $\varphi_{1}$ and $\varphi_{2}$. The above variational formulation involves an area integration along with boundary integrations. It is seen that associated with the area integration are the two equilibrium equations which, as mentioned earlier, are satisfied automatically because of the use of the stress potentials. Then the area integration in Eq. (23) vanishes and the variational formulation involves boundary integrations only which can be written, in an expanded form, as:

$$
\begin{aligned}
& \delta \Pi_{s n l}=\int_{x}^{x_{f}}\left\{\left[\sigma_{y}\left(x, \frac{W}{2}\right)-\bar{\sigma}_{d f}\right] \delta v+\left[\tau_{x y}\left(x, \frac{W}{2}\right)-\bar{\tau}_{d f}\right] \delta u\right\} d x \\
& \left.+\int_{y_{f}}^{r}\left\{\left[\sigma_{x}\left(-\frac{L}{2}, y\right)-\bar{\sigma}_{f k}\right] \delta \boldsymbol{\delta}+\left[\tau_{x y}\left(-\frac{L}{2}, y\right)-\bar{\tau}_{f k}\right\} \mathcal{V}\right\}\right\} d y \\
& -\int_{x_{t}}^{x_{h}}\left\{\left[\sigma_{y}\left(x,-\frac{W}{2}\right)-\bar{\sigma}_{R h}\right] \mathcal{\delta v}+\left[\tau_{x y}\left(x,-\frac{W}{2}\right)-\bar{\tau}_{g h}\right] \delta u\right\} d x
\end{aligned}
$$

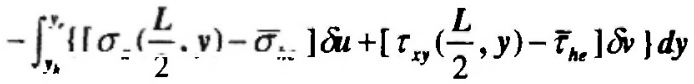

$$
\begin{aligned}
& +\int_{0}^{2 \pi}\left\{\sigma_{r}(a, \theta) \delta u_{r}+\tau_{r \theta}(a, \theta) \delta u_{\theta}\right\} a d \theta \\
& =0
\end{aligned}
$$

It is seen that the expressions in the boundary integrations in Eq. (24) are actually the boundary

$$
\begin{aligned}
& \Pi_{n=1}=\frac{1}{2} \int_{l 2}\left\{\dot{A}_{11}\left(\frac{\partial u}{\partial x}\right)^{\prime}+2_{A_{12}} \frac{\partial u}{\partial x} \frac{\partial v}{\partial y}+A_{22}\left(\frac{\partial v}{\partial y}\right)^{\prime}\right. \\
& \left.\left.+2\left(A_{16} \frac{\partial u}{\partial x}+A=\frac{\partial v}{\partial y}\right) \frac{\partial u}{\partial y}+\frac{\partial y}{\partial x}\right)+\frac{\alpha}{2} \ldots\left(\frac{\partial u}{\partial y}+\frac{\partial v}{\partial x}\right)^{3}\right\} d x d y \\
& -\int_{f_{f}}^{x_{f}}\left[\bar{\sigma}_{e f}(x) v\left(x, \frac{W}{2}\right)+\bar{\tau}_{f f}(x) u\left(x, \frac{W}{2}\right)\right] d x-\int_{J_{f}}^{y_{k}}\left[\bar{\sigma}_{f g}(y) u\left(-\frac{L}{2}, y\right)+\bar{\tau}_{f f}(y) v\left(-\frac{L}{2}, y\right)\right] d y \\
& \left.+\int_{x_{z}}^{x_{h}}\left[\bar{\sigma}_{g h}(x) v\left(x,-\frac{W}{2}\right)+\bar{\tau}_{g h}(x) u\left(x,-\frac{W}{2}\right)\right] d x+\int_{y_{h}}^{v} \overline{\sigma_{h e}}(y) u\left(\frac{I}{2}, y\right)+\bar{\tau}_{h e}(y) v\left(\frac{L}{2}, y\right)\right] d y
\end{aligned}
$$


conditions along the interior and exterior edges, as written in Eqs. (6 7). This indicates that the vanishing condition of Eq. (24), which results in the minimisation of the energy functional in Eq. (22), will give the solutions to the problem of the unloaded hole case.

For the pin-loaded hole case, the compressive stress caused by the pin load along the hole edge needs to be considered. Thus the energy functional for this case can be established by adding a boundary integration, accounting for the boundary condition in Eq. (8), to the functional in Eq. (22). This new functional can be written as:
It is seen that the vanishing condition of Eq. (26), which results in the minimisation of the energy functional in Eq. (25), satisfies the boundary conditions in Eqs. (6 and 8) under the variational statement. That is, Equation (26) is the variational formulation that gives the correct solution to the pin-loaded hole case. It is also noted that the variational formulations for both the unloaded and pin-loaded hole cases are equivalent to the minimisation of the total potential energy of the systems. Theoretically this feature will result in stable and convergent numerical computations.

$$
\begin{aligned}
& \boldsymbol{T}_{\text {pin }}=\frac{1}{2} \mathrm{f}_{\Omega}\left\{\dot{\boldsymbol{A}}_{11}\left(\frac{\partial u}{\partial x}\right)^{\prime}+\tilde{z}_{\dot{A}_{12}} \frac{\partial u}{\partial x} \frac{\partial v}{\partial y}+\dot{A}_{22}\left(\frac{\partial v}{\partial y}\right)^{\prime}\right. \\
& \left.+2\left(A_{16} \frac{\partial u}{\partial x}+A_{26} \frac{\partial v}{\partial y}\right)\left(\frac{\partial u}{\partial y}+\frac{\partial y}{\partial x}\right)+A_{s e}\left(\frac{\partial u}{\partial y}+\frac{\partial v}{\partial x}\right)^{2}\right\} d x d y \\
& -\int_{x_{f}}^{x_{f}}\left[\bar{\sigma}_{e f}(x) v\left(x, \frac{w}{2}\right)+\bar{\tau}_{e f}(x) u\left(x, \frac{w}{2}\right)\right\rfloor d x-\int_{y_{f}}^{y_{f}}\left(\sigma_{f g}(y) u\left(-\frac{L}{2}, y\right)+\tau_{f g}(y) v\left(-\frac{L}{2}, y\right)\right] d y
\end{aligned}
$$

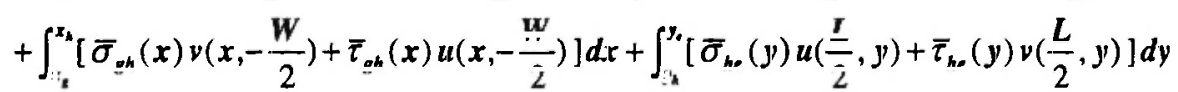

$$
\begin{aligned}
& +\int_{\theta_{1}}^{\theta_{2}} \bar{\sigma}_{o}(\theta) u_{r}(a, \theta) a d \theta
\end{aligned}
$$

where the last integration is the additional term to the functional in Eq. (22) in view of the radial compression caused by the pin load.

Similar to the discussion for the unloaded hole case, the first order variation of the functional in Eq. (25) results in a formulation for the pin-loaded hole case which involves boundary integrations only and can be written as:

$$
\begin{aligned}
& \delta \Pi_{\min }=\int_{x,}^{x_{1}}\left\{\left[\sigma_{v}\left(x, \frac{W}{2}\right)-\bar{\sigma}_{e f} \mid \mathcal{\delta} v+\left[\tau_{x y}\left(x \cdot \frac{\underline{w}}{2}\right)-\bar{\tau}_{p j} \mid \delta u\right\} d x\right.\right. \\
& +\int_{y_{f}}^{y_{x}}\left\{\left[\sigma_{x}\left(-\frac{L}{2}, y\right)-\bar{\sigma}_{f R}\right] \delta \boldsymbol{u}+\left[\tau_{x y}\left(-\frac{L}{2}, y\right)-\bar{\tau}_{f k} \mid \delta v\right\} d y\right. \\
& -\int_{x_{g}}^{\mathrm{x}_{h}}\left\{\mathrm{i} \sigma_{y}\left(x,-\frac{\boldsymbol{W}}{2}\right)-\bar{\sigma}_{R h} \mathrm{j} \hat{\sigma} \hat{v}+\left[\tau_{x y}\left(x,-\frac{\boldsymbol{W}}{2}\right)-\bar{\tau}_{g h}\right] \delta \boldsymbol{d}\right\} d x \\
& -\int_{y_{h}}^{y_{y}}\left\{\left[\sigma_{x}\left(\frac{L}{2}, y\right)-\bar{\sigma}_{h e}\right] \delta u+\left[\tau_{x y}\left(\frac{L}{2}, y\right)-\bar{\tau}_{h e}\right] \delta v\right\} d y \\
& \left.+\tilde{j}_{\dot{\theta},-i \pi}^{\theta} \mid \sigma_{r}(a, \theta) \delta u_{r}+\tau_{r \theta}(a, \theta) \delta u_{\theta}\right\} a d \theta \\
& +\int_{\theta_{1}}^{\theta_{i}}\left\{\left[\sigma_{r}(a, \theta)+\sigma_{a}(\theta)\right] \delta u_{r}+\tau_{r \theta}(a . \theta) \delta u_{\theta}\right\} a d \theta \\
& =0
\end{aligned}
$$

\section{TRIAL FUNCTIONS AND LAGRANGIAN MULTIPLIER}

Now the problem is to select appropriate stress potentials, $\varphi_{1}$ and $\varphi_{2}$, as the trial functions for the respective variational formulations derived. The selection of trial functions is an important step in the variational methods for accurate numerical calculations. For the convergence and efficiency of calculations in the complex theory of two-dimensional elasticity, the wellknown Laurent series is often used for the stress potentials. In this work, functions in the form of a truncated Laurent series with conformal mapping are employed for the stress potentials, $\varphi_{1}$ and $\varphi_{2}$, as:

For unloaded hole:

$$
\begin{aligned}
& \varphi_{1}=\sum_{n=1}^{N}\left(C_{1 n} \zeta_{1}^{n}+D_{1 n} \zeta_{1}^{-n}\right) \\
& \varphi_{2}=\sum_{n=1}^{N}\left(C_{2 n} \zeta_{2}^{n}+D_{2 n} \zeta_{2}^{-n}\right)
\end{aligned}
$$

For pin-loaded hole:

$$
\begin{aligned}
& \varphi_{1}=C_{11} \ln \zeta_{1}+\sum_{n=1}^{v}\left(C_{1 n} \zeta_{1}^{n}+D_{1 n} \zeta_{1}^{-n}\right) \\
& \varphi_{2}=C_{20} \ln \zeta_{2}+\sum_{n=1}^{v}\left(C_{2 n} \zeta_{2}^{n}+D_{2 n} \zeta_{2}^{-n}\right)
\end{aligned}
$$


where the undetermined constants $C_{1 \mathrm{n}}, D_{\mathrm{ln}}, C_{2 \mathrm{n}}$ and $D_{2 \mathrm{n}}$ are, in general, complex and the mapping functions are:

$$
\zeta_{k}(x, y)=\frac{z_{k} \pm \sqrt{z_{k}^{2}-a^{2}\left(1+\mu_{k}^{2}\right)}}{a\left(1-i \mu_{k}\right)}, \quad i:=1,2
$$

where $i$ is the imaginary unity and the sign of the square root term is chosen such that the hole of radius $a$ is mapped to a unit circle. Noting that the stress potentials in Eq. (28) for the pin-loaded hole case involve logarithm terms, the following conditions are imposed (as in Xiong, 1996) to ensure single-valued displacement components:

$\operatorname{Im}\left(p_{1} C_{10}+p_{2} C_{20}\right)=0, \quad \operatorname{Im}\left(q_{1} C_{10}+q_{2} C_{20}\right)=0$

The complex constants in the trial functions are to be determined by the variational formulations derived and the number of constants is $2 N$ and $2 N+1$ for unloaded hole and pin-loaded hole cases, respectively. Once these constants are computed, the stress and displacement components can be calculated using Eqs. (18 19).

\section{RESULTS AND DISCUSSION}

To verify the analytical approach developed, several cases of a laminate with a single unloaded and pinloaded hole have been analysed. The problem is a square AS4/3501-6 [45/0/-45/90] $]_{6 s}$ laminate with a centrally located hole. The width of the plate is $W=$ $152.4 \mathrm{~mm}$. The mechanical properties of each ply are: $E_{1}$
$=140 \mathrm{GPa}, E_{2}=8.2 \mathrm{GPa}, G_{12}=6.2 \mathrm{GPa}$, and $v_{12}=0.3$. The ply thickness is $0.127 \mathrm{~mm}$. For the sake of simplicity, uniform normal and shear stresses are applied along the exterior edges. These applied loads are:

For unloaded hole case.

$$
\begin{aligned}
& \sigma_{e f}=\bar{\sigma}_{g h}=1 \mathrm{~N} / \mathrm{m}, \quad \bar{\sigma}_{f k}=\bar{\sigma}_{h e}=2 \mathrm{~N} / \mathrm{m} \\
& \bar{\tau}_{e f}=\bar{\tau}_{f r}=\bar{\tau}_{g h}=\bar{\tau}_{h e}=0.5 \mathrm{~N} / \mathrm{m}
\end{aligned}
$$

For pin-loaded hole case.

$$
\begin{aligned}
& \bar{\sigma}_{e f}=2 \mathrm{~N} / \mathrm{m}, \bar{\sigma}_{f h}=1 \mathrm{~N} / \mathrm{m}, \bar{\sigma}_{\mathrm{gh}}=1 \mathrm{~N} / \mathrm{m}, \bar{\sigma}_{h e}=2 \mathrm{~N} / \mathrm{m}, \\
& \bar{\tau}_{e f}=\bar{\tau}_{f k}=\bar{\tau}_{g h}=\bar{\tau}_{h e}=0.5 \mathrm{~N} / \mathrm{m}
\end{aligned}
$$

The complex variational approach developed involves some numerical calculations. Therefore, an analysis was conducted for both unloaded and loaded hole cases to examine the convergence characteristics of the approach. A central hole with a radius of $25.4 \mathrm{~mm}$ was considered and the highest stress components along the hole edge were calculated with an increasing number of terms in the stress potentials. The results versus the $N$ values are presented in Table 1 and Table 2 for unloaded and loaded hole cases, respectively. It is shown that the numerical calculations are stable and converged for the selected range of $N$ values. Higher $N$ values may result in divergence due to round-off errors in numerical calculations, although the computations are theoretically convergent. In the following examples, it has been set that $N=9$ for both unloaded hole and pinloaded hole cases.

Table 1

Stress components around hole edge $(n / m)$ in unloaded case.

\begin{tabular}{|c|c|c|c|c|c|c|c|c|}
\hline $\mathbf{N}$ & 3 & 4 & 5 & 6 & 7 & 8 & 9 & 10 \\
\hline$\left(\sigma_{\theta}\right)_{\max }$ & 6.995 & 7.078 & 7.099 & 7.128 & 7.436 & 7.428 & 7.630 & 7.615 \\
\hline$\left(\sigma_{r}\right)_{\max }$ & -0.504 & -0.496 & 0.077 & 0.078 & 0.043 & 0.041 & 0.022 & 0.019 \\
\hline$\left(\tau_{r g}\right)_{\max }$ & -1.178 & -1.188 & -0.134 & -0.133 & -0.055 & -0.056 & -0.019 & -0.017 \\
\hline $\boldsymbol{P}_{f}(\mathbf{N})$ & 0.0017 & 0.0012 & 0.0014 & 0.0011 & .0011 & .0011 & 0.0011 & 0.0011 \\
\hline
\end{tabular}


Table 2

Stress components around hole edge $(n / m)$

in pin-loaded case.

\begin{tabular}{|c|c|c|c|c|c|c|c|c|}
\hline $\mathbf{N}$ & 3 & 4 & 5 & 6 & 7 & 8 & 9 & 10 \\
\hline$\left(\sigma_{\theta}\right)_{\max }$ & 10.657 & 10.621 & 10.756 & 10.760 & 10.865 & 10.863 & 11.070 & 11.063 \\
\hline$\left(\sigma_{r}\right)_{\max }$ & -5.654 & -5.666 & -5.397 & -5.380 & -5.364 & -5.369 & -5.344 & -5.354 \\
\hline$\left(\tau_{r \theta}\right)_{\max }$ & -1.249 & -1.230 & -0.203 & -0.206 & -0.120 & -0.126 & 0.077 & 0.072 \\
\hline $\boldsymbol{P}_{f}(\mathrm{~N})$ & 0.2167 & 0.2169 & 0.2156 & 0.2156 & 0.2156 & 0.2155 & 0.2155 & 0.2157 \\
\hline$\varepsilon$ & $1.27 \%$ & $1.09 \%$ & $1.05 \%$ & $0.07 \%$ & $0.05 \%$ & $0.03 \%$ & $0.03 \%$ & $0.09 \%$ \\
\hline
\end{tabular}

Closed-form solutions for infinite notched laminated plates (Lekhnitskii, 1968) with a superposition technique were used to verify the present approach for an unloaded hole under the loads in Eq. (31). The radius of the hole is $5 \mathrm{~mm}$ which is small compared to the plate width so that the plate can be assumed to be infinitely wide. The hoop stress distribution around the hole from the present approach is shown in Figure 2 and compared with the closed-form solutions. The good agreement obtained verifies the accuracy of the present approach for analysing infinite notched plates.

To verify the accuracy of the present approach for analysing finite laminated plates with unloaded and pinloaded holes, finite element analyses using $\mathrm{MSC} /$

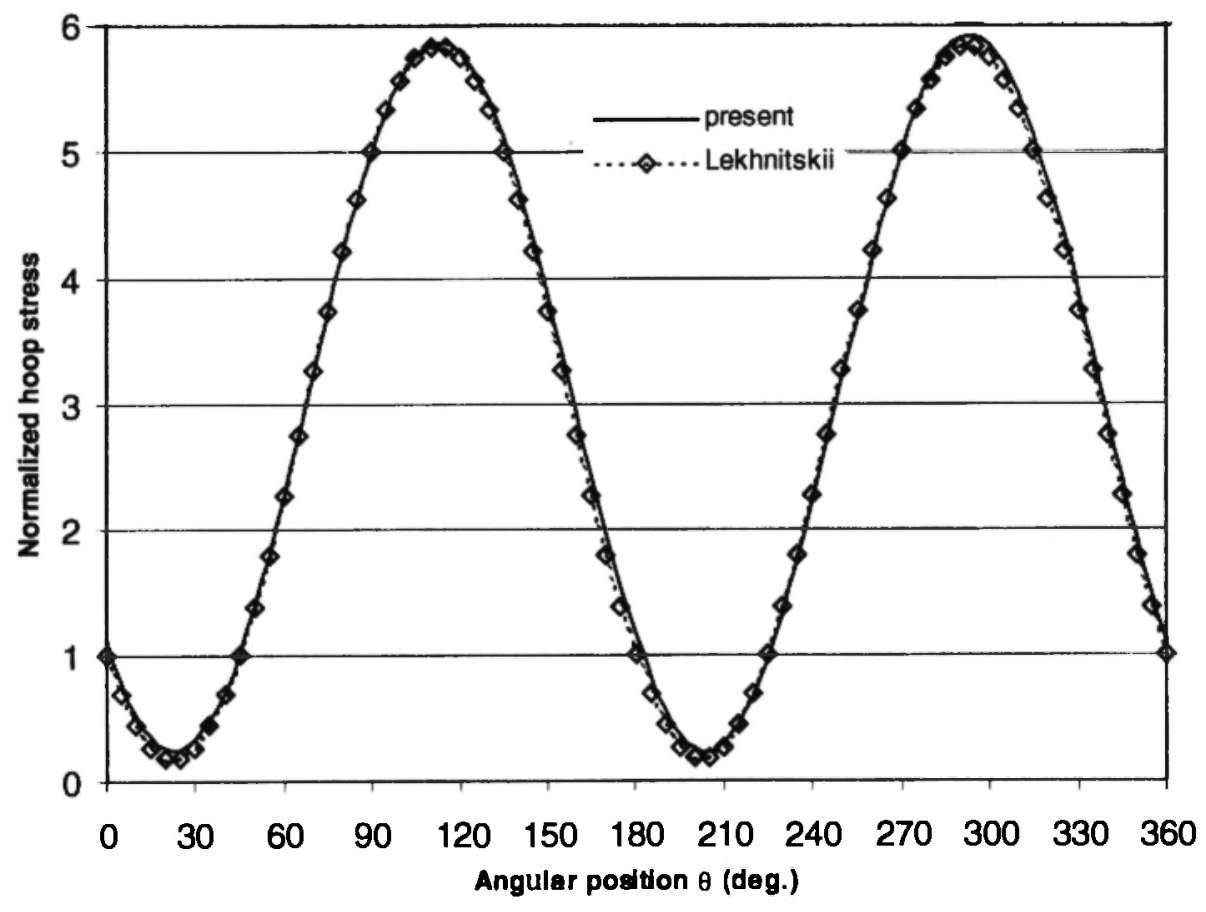

Fig. 2: Hoop stress around unloaded hole in infinite plate. 
NASTRAN were conducted for various loading cases and hole sizes. Comparisons of results between the present approach and the finite element analyses are made and shown in Figures 3 6. It is seen that the accuracy of the present approach is comparable to that of the finite element analyses using a very fine mesh with 200 elements (CQUAD4) around the hole edge and thus the efficiency of the present approach is demonstrated.

Figure 3 shows the hoop stresses from the present approach and the finite element analysis for an unloaded hole with a radius of $25.4 \mathrm{~mm}$ under the loads in Eq. (31). It is seen that the peak hoop stresses occur at locations of $\theta=115^{\circ}$ and $\theta=295^{\circ}$ due to the effect of the shear load as compared to the locations of $\theta=90^{\circ}$ and $\theta=270^{\circ}$ under a uni-directional load in $x$-direction. The peak stresses around an unloaded hole of different diameters are presented in Figure 4 and the significant effects of the hole size for a fixed plate width on the stress concentration are shown.

Figure 5 shows the hoop and bearing stresses from the present approach and the finite element analysis for a pin-loaded hole with a radius of $25.4 \mathrm{~mm}$ under the loads in Eq. (32). In this loading case, the peak hoop stresses occur at locations of $\theta=135^{\circ}$ and $\theta=315^{\circ}$, while the peak bearing stress occurs at the location of $\theta$ $=225^{\circ}$. Similarly, the peak stresses around a pin-loaded hole of different diameters are presented in Figure 6 and the significant effects of the hole size for a fixed plate width on the stress concentration are shown.

\section{CONCLUSIONS}

An analytical approach based on complex variational formulations has been developed for the stress analysis of composite plates containing an unloaded or a pin-loaded hole under general in-plane loads. The accuracy and efficiency of the analytical approach have been verified against the closed-form solutions for an infinite notched plate and the finite element results for plates with finite width. The salient features of the approach developed include:

1) analytical expressions can be obtained for calculating stress and displacement components at any locations,

2) plates with any size of the hole diameter can be analysed because the finite size effects are automatically taken into account in the formulations, and

3) any combination of in-plane loads can be dealt with.

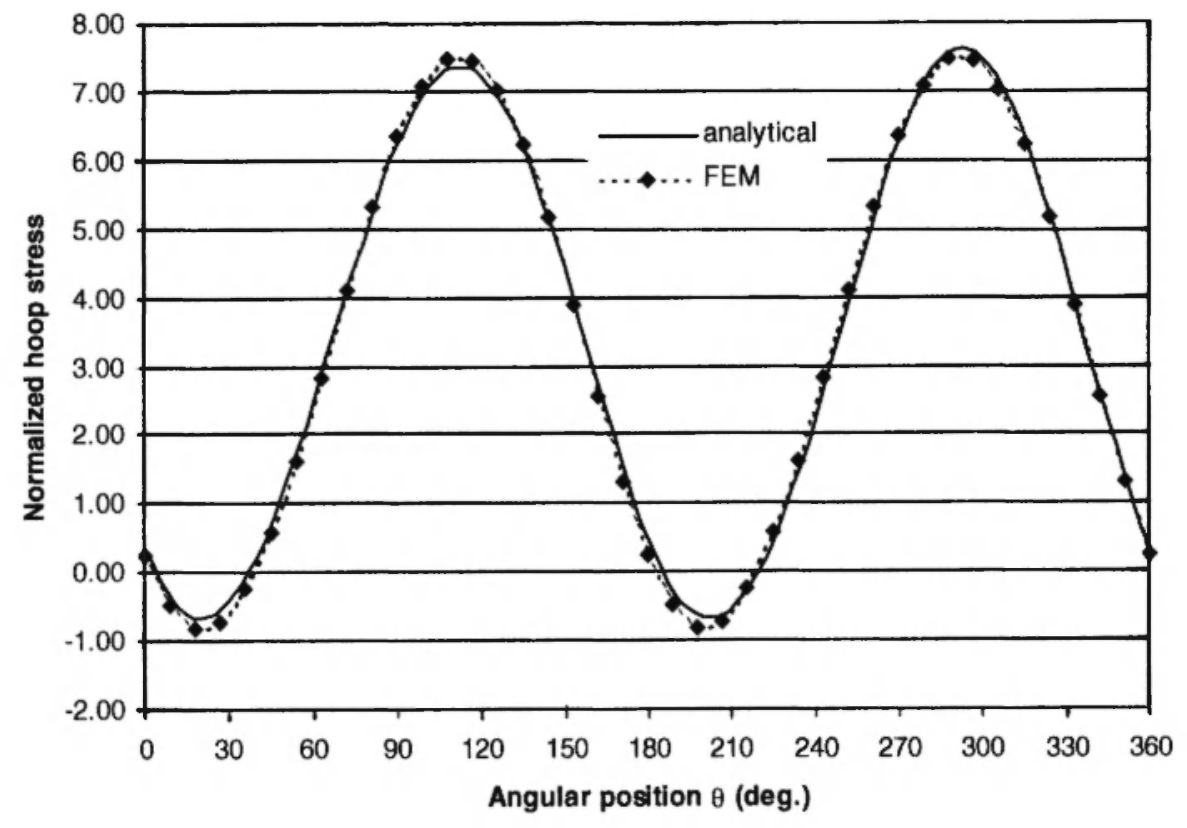

Fig. 3: Hoop stress around unloaded hole in finite plate. 


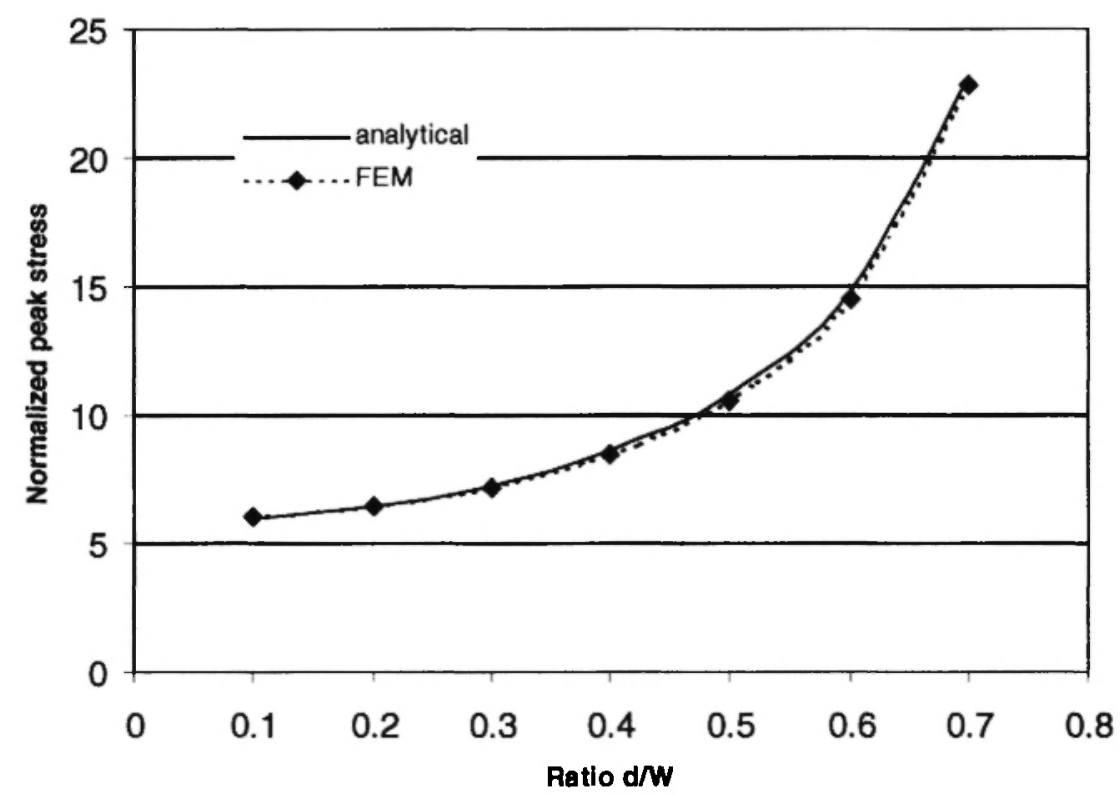

Fig. 4: Peak stress around unloaded hole of various diameters.

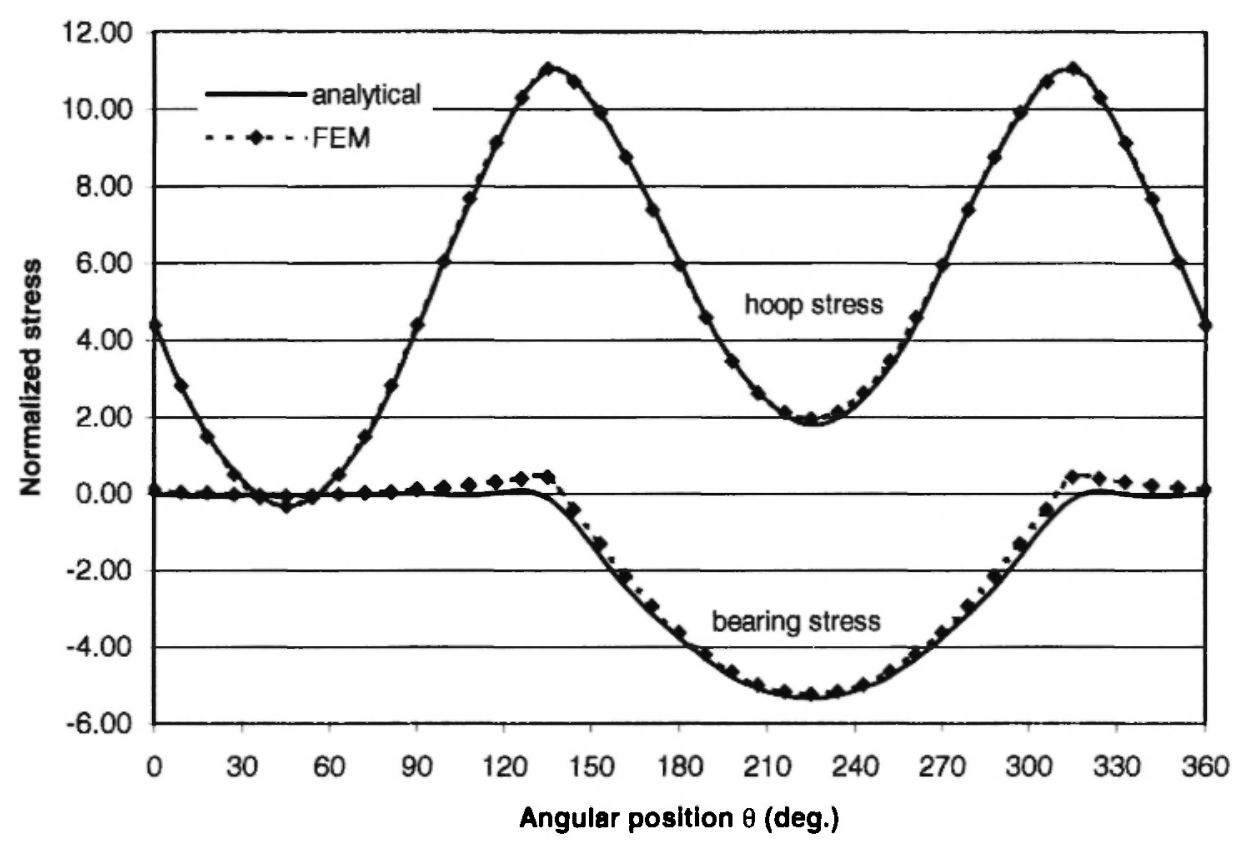

Fig. 5: Hoop and bearing stresses around pin-loaded hole. 


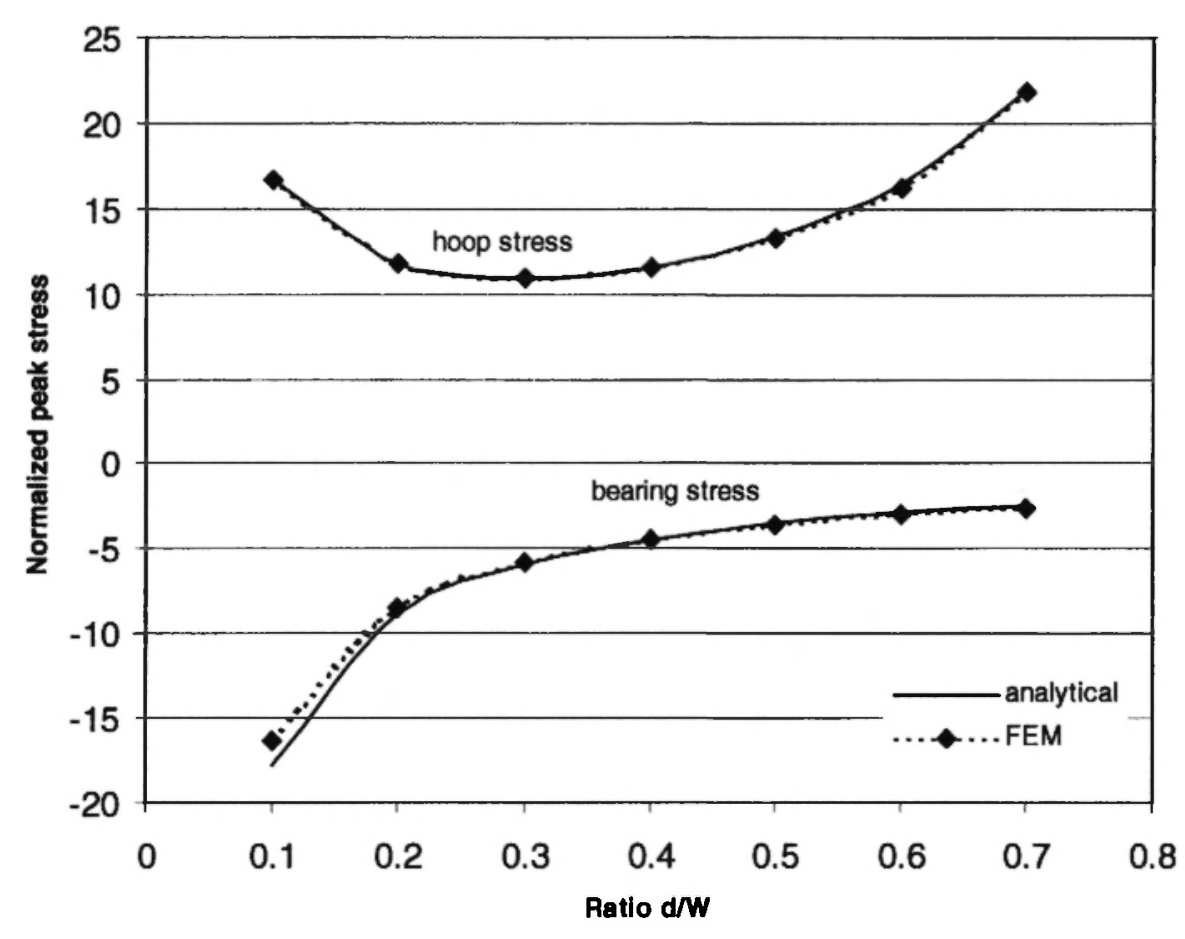

Fig. 6: Peak stresses around pin-loaded hole of various diameters.

The results obtained indicate that both the shear load and the hole size have significant effects on the magnitude and locations of the stress concentrations around the hole. Consideration of these effects is very important in investigating the failure characteristics of composite laminates with unloaded and loaded holes.

\section{ACKNOWLEDGEMENT}

This work has been carried out under lAR Program 3G3, Aerospace Structures, Structural Dynamics and Acoustics, Project/Subproject SAA-00 Computational Structures Technology Development and Project/Subproject JGM-02 Mechanical Joining Technology in Composites. The financial support provided by the Department of National Defence of Canada and the comments made by Dr. Osama Bedair are gratefully acknowledged.

\section{REFERENCES}

1. O.L. Bowie and D.M. Neal. A modified mappingcollocation technique for accurate calculation of stress intensity factors, Int. J. Fracture Mechanics, 6, 199-206 (1970).

2. Fu-Kuo Chang, R.A. Scott and G.S. Springer. Strength of bolted joints in laminated composites, AFWAL-TR-84-4029 (1984).

3. J.H. Crews, Jr., C.S. Hong and I.S. Raju. Stressconcentration factors for finite orthotropic laminates with a pin-loaded hole, NASA Technical Paper 1862 (1981).

4. Th. De Jong. Stresses around pin-loaded holes in elastically orthotropic or isotropic plates, Journal of Composite Materials, 11, 313-331 (1977).

5. S.P. Garbo and J.M. Ogonowski. Effect of variances and manufacturing tolerances on the design strength and life of mechanically fastened composite joints, I. Methodology development and data evaluation, AFWAL-TR-81-3041 (1981). 
6. S.G. Lekhnitskii. Anisotropic Plates, Gordon and Breach Science Publishers, New York, 1968.

7. E. Madenci and L. Ileri. Analytical determination of contact stresses in mechanically fastened composite laminates with finite boundaries, Int. J. Solids Structures, 30, 2469-2484 (1993).

8. D.W. Oplinger. Bolted joints in composite structures - an overview, AGARD Conference Proceedings 590: 'Bolted'Bonded Joints in Polymeric Composites, NATO, France, 1996.

9. J.P. Waszczak and T.A. Cruse. Failure mode and strength predictions of anisotropic bolt bearing specimens, Journal of Composite Materials, 5, 421-425 (1971).

10. Y. Xiong. An analytical method for failure prediction of multi-fastener composite joints, International Journal of Solids and Structures, 33, 4395-4409 (1996).

11. K.D. Zhang and C.E.S. Ueng. Stresses around a pin-loaded hole in orthotropic plates with arbitrary loading direction, Composite Structures, 3, 119-143 (1985). 
